\title{
STRESS ZONES IN FOETAL ARTERIES
}

\author{
BY \\ J. H. ROBERTSON \\ From the Department of Pathology, Queen's University, Belfast
}

(RECEIVED FOR PUBLICATION JULY 28, 1959)

\begin{abstract}
In a study of the peripheral arteries of foetuses it was found that focal areas of intimal thickening and of splitting of the internal elastica had developed, the thickening being similar to that found in the coronary arteries. Characteristically the thickenings were found in the mouths of branches and in those vessels subject to pulsation. It is believed that they are physiological in nature and a response of the vascular wall to the stresses produced by focal areas of impaired pulsation and not degenerative as formerly thought.

The evolution of these arterial cushions is traced from early foetal life; their most important constituent is bundles of medial longitudinal muscle. Both thickening and splitting of the internal elastica are brought about by a radial reorientation and migration of the muscle cells of the media.
\end{abstract}

\section{Material}

The vessels were obtained from 25 foetuses, of which 14 were male and 11 female. Their maturity ranged from 30 weeks' gestation to full term. All of them died within 24 hours of birth except one, a premature infant of 35 weeks' gestation who lived for four days after birth. In addition, the vessels of four early foetuses of from 14 to 20 weeks' maturity were also examined.

A small segment of each artery studied was taken from the same site in each case. In the upper limb these comprised the brachial artery, both at the level of the insertion of the deltoid muscle and in the antecubital fossa, as well as the radial artery at the wrist. In the leg, the vessels examined were the femoral artery, in the upper part of the subsartorial canal, the popliteal, in the popliteal fossa, and the posterior tibial artery from just above the medial malleolus.

From each of these short segments three blocks were taken more or less at random, although, if a branch were present, then its mouth was included in one of the blocks. Several sections were also taken from the abdominal aorta in each case to show the mouths of the lumbar vessels. In eight cases random blocks were taken from the coronary arteries for comparative purposes. All the sections were stained with haematoxylin and eosin and with Weigert's elastic tissue stain and van Gieson. Some were also stained with Sudan IV and haematoxylin for lipoid.

\section{Findings}

Peripheral Arteries.-In most of the peripheral vessels the intima consisted only of a layer of endothelium lying directly on the internal elastic lamina. In some, however, there were focal areas of thickening which varied from a simple splitting of the internal elastic lamina to the formation of large cushion-like structures which projected into the lumen.

These arterial cushions had a characteristic distribution in the peripheral vessels as well as a distinctive location in their walls. They were found only in the popliteal and lower end of the brachial artery. Most often they occurred at the margins of branches and in the wall of the parent vessel opposite the mouth of the branch (Fig. 1). However, they were not restricted to these sites and, especially in the popliteal artery, they were also found in places where there was no evidence of branching. Nevertheless, there tended to be a pattern in their distribution in that they often occurred in groups, several being present in one segment of the vessel. There was a marked difference both in the size and frequency of cushions in the popliteal and brachial arteries. They were found in the popliteal vessel in $76 \%$ of the cases and here attained a much larger size than they did in the lower end of the brachial artery where they were also less frequent.

The structure of the cushions was variable, but they were all musculo-elastic in nature, were generally symmetrical in shape and formed a definite thickening of the vessel wall. The great majority of them had both an intimal and a medial component. In the media there occurred 


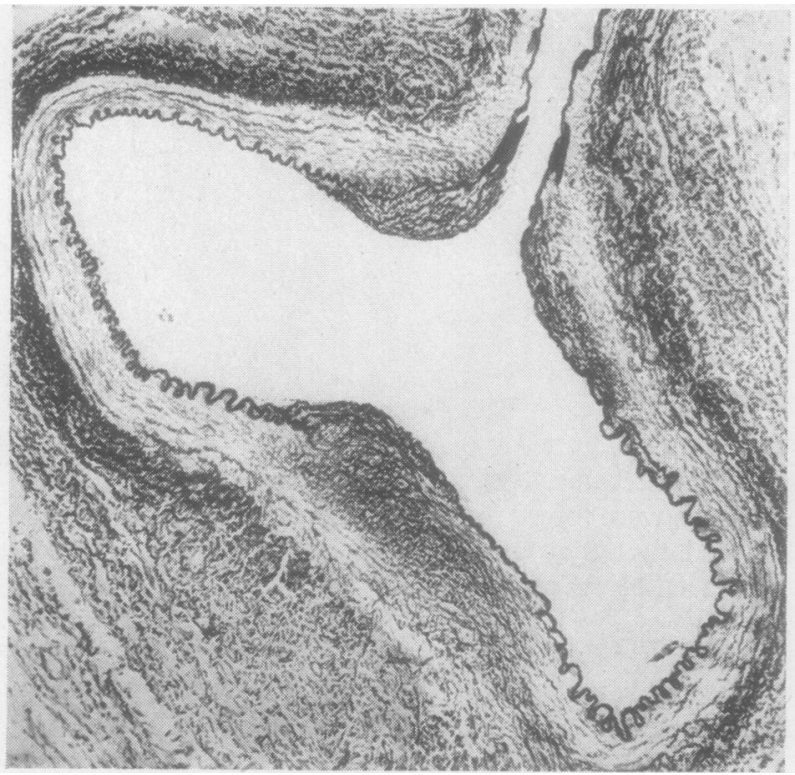

Fig. 1.-Popliteal artery in a female at full term, showing the characteristic location of arterial cushions. Weigert, $\times 70$.

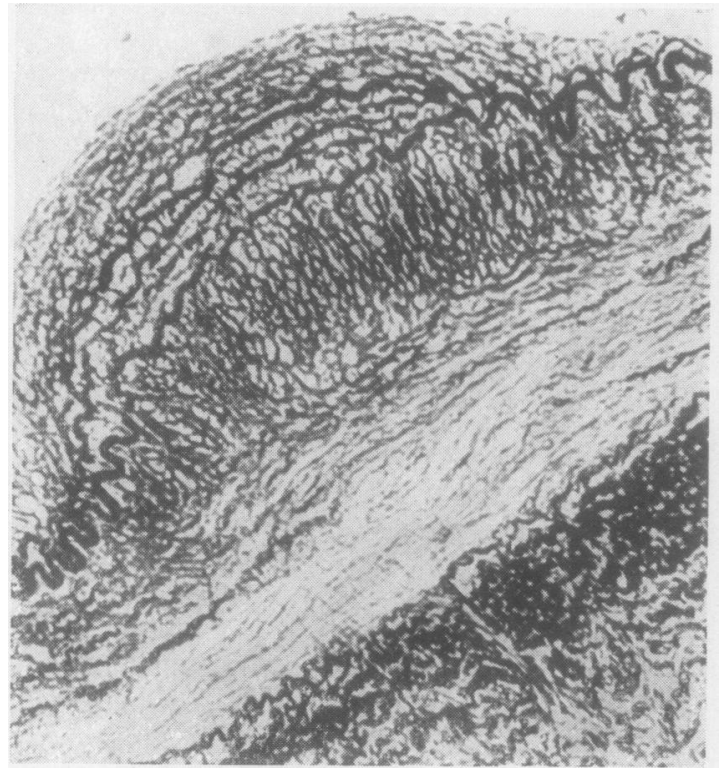

FIG. 3.-Popliteal artery in a male, full term. The internal elastica can be identified. Below it the elastic network of a medial longitudinal muscle band is prominent. In the intima longitudinal muscle lies in an elastic mesh derived from the elastica. Weigert, $\times 250$.

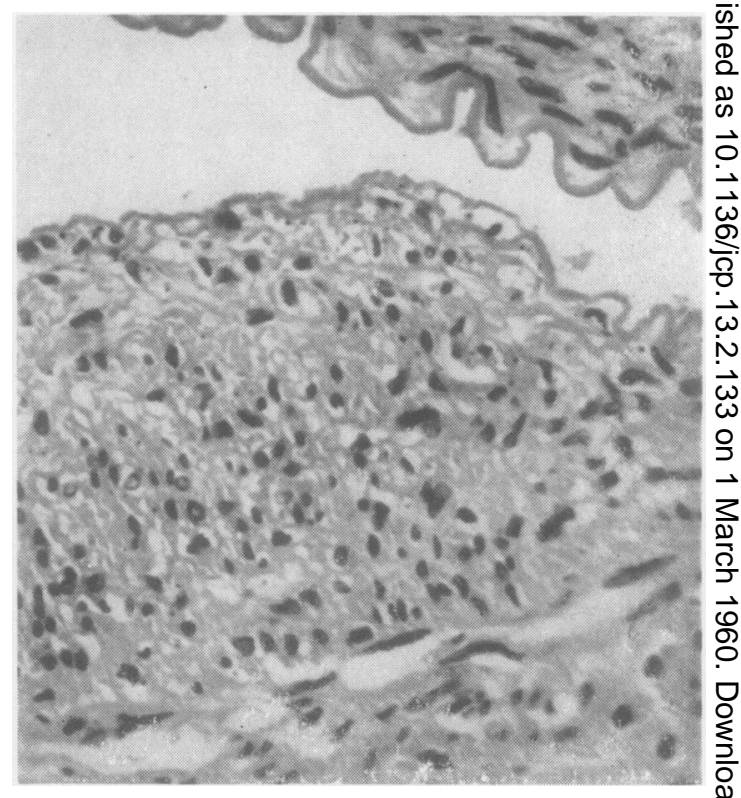

FIG. 2.-Popliteal artery in a female of 30 weeks' gestation. cushion showing the longitudinal direction of its muscle. Haematoxylin and eosin, $\times 350$.

FIG. 4.-Popliteal artery in a female, full term. The internal elastico has become greatly interrupted and lies beneath a mound of elastic fibrils and longitudinal muscle. A thin rim of longi tudinal medial fibres can be seen. Weigert, $\times 175$. 
bundles of longitudinally running muscle fibres which lay in a feltwork of fine elastic filaments so intimate that almost every muscle fibre was invested by it (Figs. 2 and 3). The intimal portion was formed by a splitting of the internal elastic lamina, sometimes in a fibrillary fashion and sometimes into several coarse lamellae between which longitudinal muscle was also present (Fig. 3). In occasional cushions the intimal element was predominant so that remnants of the internal elastica could be clearly seen beneath a large mound of longitudinal muscle and elastic tissue, while in the media only a thin rim of longitudinal fibres could be distinguished (Fig. 4). More often, however, instances were found in which a medial component alone was present. This took the form of a short band of longitudinal muscle which produced no thickening of the wall and lay immediately below an internal elastic lamina which was intact or showed only the earliest stages of splitting (Fig. 5). Transitions between these forms and the more complex cushions frequently occurred.

Although, as described, these cushions were mainly composed of longitudinally running muscle some radially directed fibres were also observed. These occurred especially on the deep surface of a cushion and in places where longitudinal muscle bundles in the media appeared to be forming and extending laterally in the vessel wall. Here the circular fibres of the media could be seen to sweep round in a radial fashion and to merge with the longitudinal muscle. Quite often also, radial fibres were seen which appeared to be squeezing themselves through small gaps in the internal elastic lamina.

It is emphasized that the cushions described above were essentially musculo-elastic structures and that the vast majority of them showed either no evidence of a fibrous tissue component or contained but a few strands of it running alongside the elastic fibrils of the thickening. Only occasional cushions were quite definitely fibrous. In these the elastic tissue tended to be more finely divided and the fibrous tissue occurred in the superficial parts of the thickening amongst the mesh of fine elastic filaments.

Deposits of lipoid were not a characteristic feature of these arterial cushions. In seven of the cases serial frozen sections of the popliteal artery were stained with Sudan IV and haematoxylin for lipoid. In this way, three or four different cushions from each case were studied. In only one, a premature infant of 35 weeks' gestation who survived for four days after birth, was any

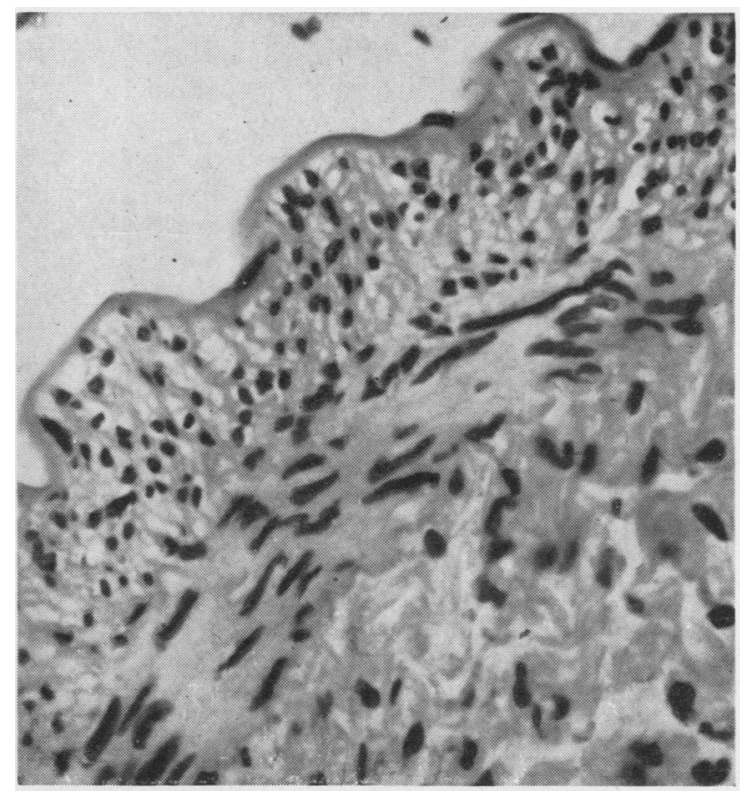

FIG. 5. -A band of longitudinal muscle lies below the internal elastica which shows no evidence of reduplication. Haematoxylin and eosin, $\times 400$.

lipoid detected. In this case every cushion was involved, and the lipoid occurred as a fine, diffuse deposit in the superficial parts of the thickenings. In the sections stained with haematoxylin and eosin the appearance of only three cushions suggested that they might contain lipoid. In only one of them was there any fibrous tissue, the other two being purely musculo-elastic in structure.

Aorta.-In contrast to the peripheral vessels the aorta showed little evidence of the formation of muscular thickenings at the margins of its branches. In the section stained with Weigert's elastic stain there was no evidence of complex elastic tissue changes similar to those which threw the cushions of the peripheral arteries so readily into relief. A close examination of the sections stained with haematoxylin and eosin, however, did show that there was a definite tendency for the muscle cells in the aortic wall to be longitudinally arranged at the margins of branches. In comparison with the changes in the peripheral arteries those in the aorta were quite unspectacular and were present at the mouths of only a proportion of the branches.

Arteries of the Early Foetus.-In an attempt to trace the earliest beginnings of arterial cushions, the peripheral vessels of four early foetuses of $14,16,18$, and 20 weeks' gestation 
were examined. The 16-week foetus was the earliest one in which there was a suggestion of the formation of muscular cushions. They occurred as slight thickenings of the wall of the popliteal artery which increased in both size and number during the eighteenth and twentieth weeks. Their structure was at first simple and consisted solely of a small node of longitudinally orientated muscle with no evidence of reduplication of the overlying internal elastic lamina. It was not until the twentieth week that this lamina split. At this time too, the longitudinal muscle of the thickening began to develop its intrinsic elastic net (Fig. 6).

Coronary Arteries. - In view of previous reports on the finding of intimal cushions in the coronary arteries of the newborn, it was thought necessary to compare the thickenings already described with those occurring in these vessels. Accordingly, the coronary arteries were examined in eight cases. These comprised six male and two female foetuses, all of which were stillborn save one infant who survived for four days after birth. Random sections were taken from both the
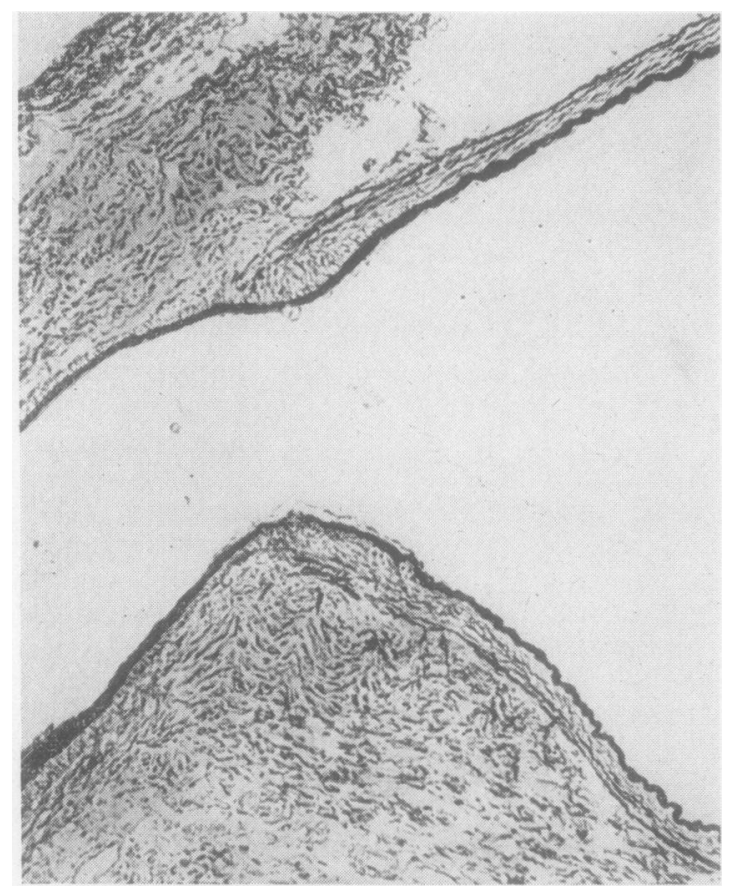

FIG. 6.-Popliteal artery in a 20-week foetus. Bundles of longitudinal muscle are present at the margins of a branch. The internal elastica shows early reduplication. The gap in the lamina over the left cushion is occupied by radial smooth muscle cells. Weigert, $\times 125$. anterior descending and the circumflex branch of the left coronary artery.

In four of the infants, the two female and two of the male, no coronary cushions were found. In the other four structural changes were present $\bar{c}$ which were similar to those found in the $\vec{\Phi}$ peripheral arteries. They differed from those in the popliteal artery only in that they were $\infty$ generally smaller and more widely scattered in $\overrightarrow{0}$ the coronary vessels. The only coronary cushion $\overrightarrow{\vec{A}}$ in which lipoid was found came from the heart of $\omega$ the premature infant of 35 weeks' gestation who lived for four days after birth. There was no? evidence in the van Gieson stain of a connective $\vec{\omega}$ tissue component in this cushion. It is interesting to note that it was this same infant which was the $\vec{\omega}$ only one to show the presence of a diffuse 0 infiltration of lipoid in the frozen sections of the popliteal cushions.

\section{Discussion}

In 1946, Dock reported the finding of intimal cushions in the coronary arteries of the newborn, but did not go into any histological detail. He found that the thickenings were more marked in $\frac{\partial}{\partial}$ male than female infants, and considered that their presence was perhaps related to the coronary $\mathbb{D}$ vessels having to adapt themselves to a changing $\overrightarrow{\vec{D}}$ length during cardiac systole. Hence, the males, with their slow pulses, high stroke volume, and greater changes in length of the vessels, were more severely affected. Dock believed that thus was established the predilection of atherosclerosis for 8 the coronary vessels and its greater incidence in males than females.

The presence of these cushions in the coronary $\stackrel{0}{0}$ arteries of infants was later confirmed by $₹$ Fangman and Hellwig (1947), Lack (1947, cited by 옹 Anderson, 1953), Minkowski (1947), Levene $\supset$ (1956), and Moon (1957). However, these later authors differed from Dock in the interpretation $N$ of their significance. Thus, Fangman and Hellwig believed that they represented the earliest $\stackrel{N}{\circ}$ stages of arteriosclerosis, that a deposition of $\underset{\omega}{N}$ lipoid was the primary event in their formation and that this then resulted in a degeneration of 0 elastic tissue. Both Levene and Moon also $\stackrel{\overparen{D}}{\overparen{D}}$ believed these coronary cushions to be patho- $\stackrel{\oplus}{+}$ logical. The former held that they represented $T$ sites of underlying medial weakness and the latter that they were the early phases of the arterio- $\stackrel{\mathbb{Q}}{\stackrel{\oplus}{\oplus}}$ sclerotic process. Minkowski, on the other hand, $\mathbb{\perp}$ was more cautious. While recognizing that the thickenings showed a strong resemblance to early 
adult arteriosclerosis, he considered that their real significance had yet to be clarified. Lack is stated by Anderson to have found that the thickenings were confined to points of branching tapering off above and below these levels, but no opinion is given as to their nature.

The findings of the present study do not support the view that these cushions are either primarily intimal in origin or degenerative in nature. Thus, as has been described, from their inception in early foetal life and throughout their development, the most important constituent of these thickenings is a bundle of longitudinal medial muscle. Indeed, when first formed, they consist solely of longitudinal muscle. It is only during their later development that there is a progressive splitting of the overlying internal elastica and the acquirement, by a proportion, of connective tissue and occasional foam cells. The very appearance of the cushions is opposed to the theory that they are degenerative in nature. There is a symmetry and even a suggestion of architectural design in the interweaving of their smooth muscle and elastic tissue which suggest purpose and function.

Previous workers have stressed the similarity in the distribution of the cushions in the coronary arteries to that of atherosclerosis and have put this forward as further evidence of their close relationship to this disease. However, when their presence also in the popliteal and antecubital fossae and their peculiar tendency to localize in certain situations in these vessels is considered, then a different explanation of their significance becomes probable.

In attempts to explain the tendency of arteriosclerosis to localize at branchings, it has long been suggested that pulsation gives rise to important haemodynamic stresses in these regions (Duguid, 1926; Moschcowitz, 1942). Duguid especially has emphasized the systolic elongation in vessels during pulsation, and believed that in regions of branching the branch acted as a "drag," tending to fix the parent vessel, resulting in a damaging strain to its wall. This tendency to elongate as well as dilate during systole is often forgotten, but that it is an important effect of pulsation is evident on consideration of the fibrosed arteriosclerotic peripheral vessels of the elderly which are not only markedly dilated but are also tortuous.

If a branch does impair the systolic elongation of the parent vessel, perhaps by the tone and elasticity of its muscular coat and by the interlacing of its adventitial coat at the point of junction, then at each pulse beat there would be a tugging at the margins of the branch which would be longitudinal in direction. In the peripheral arteries, where the muscular coat is composed of circular muscle fibres, such a longitudinal stress cannot be effectively countered and would eventually be likely to lead to a disruption of the wall at this point. It is suggested, therefore, that the cushions of foetal arteries are but the physiological reaction of the vessel to such a longitudinal stress and that by the tonus and elasticity of their muscle they prevent tearing and damage to the arterial wall. The muscular cushions found in some cases opposite the mouths of branches as well as at their margins are probably due to similar causes. Here, it would seem, the interweaving of the muscular and adventitial coats of the parent vessel and branch is such that it leads to fixation of the whole segment of the parent artery and not of just the region around the orifice of the branch. In the same way, the presence of any local factor such as the proximity of an artery to bone, its crossing by a fascial band or tendon, or the presence of a neighbouring actively contracting muscle could all result in focal areas of impaired pulsation and lead to the development of cushions which were not related to the orifices of branches. Such an hypothesis also explains the anatomical distribution of these structures. Two factors are required for their presence: (1) marked pulsation of the artery and (2) focal points of fixation. It is, therefore, not surprising to find that they are prominent in the coronary arteries which lie on the surface of the heart and are tethered by the muscular branches penetrating the myocardium. Similarly, both the lower brachial and popliteal vessels are unrestricted because they lie in the popliteal and antecubital fossae and yet are fixed by the geniculate arteries and the vessels given off at the elbow joint respectively.

An interesting feature in the distribution of these muscular cushions is the observation that they are much more prominent in the lower than in the upper limbs. That gravitational forces can have played no part in this predilection is evident since the majority of the foetuses must have spent their existence in an inverted position. Hamilton and Dow (1939) have found that in the dog, where also gravity can play little part, the systolic pressure was higher, and, what is probably more important, the pulse pressure curve was much steeper in the abdominal than in the thoracic aorta. This finding suggests that the difference in the pulse wave in the lower limbs is a function, not of gravity, but of the action of the aorta, a conclusion which correlates well with the present findings. It would seem probable that it is the 
steepness of the pulse wave rather than its height which is the main factor in dictating the presence or absence of cushions. The steeper the pulse wave, the more sudden and damaging would be its effects at branchings. For this reason the absence of cushions in the tibial and radial vessels is also not unexpected, for when the pulse wave reaches these arteries it is flattened and much less steep than in the larger trunks (Sodeman, 1956).

In the present series of cases muscular cushions were not a feature of either the femoral or upper part of the brachial artery. It would seem probable that in the case of the femoral vessel this is due to the close support given to it by the muscles forming the subsartorial canal. This would uniformly damp down the pulsation around the whole of its circumference so that the stresses produced at branchings would be mitigated. The more rounded pulse wave in the arm and also the lack of any large branches to fix the upper part of the brachial artery probably dictate the absence of cushions in this vessel.

The aorta presents an apparent discrepancy in the argument put forward above, for it is in this vessel that pulsation is most marked and yet, paradoxically, cushion formation is not prominent. This, however, is also in accordance with the hypothesis, for it holds that the raison d'être of these structures is the inability of the circular muscle of the peripheral arteries adequately to resist a longitudinal stress. In contrast to the peripheral vessels, the main strength of the aorta lies in the presence of its many concentric elastic membranes. These membranes could resist a stretching force just as well as a dilating one, so that there is little need for the development of muscular cushions. Nevertheless, even in this vessel there is a tendency for the smooth muscle at the margins of branches to become longitudinally orientated.

No measurements of the relative thickness of the intima in males and females were attempted in the present study. However, Dock's finding that the male intima was thicker than the female, and his suggestion that this was due to a higher stroke volume of the male heart, is quite compatible with the hypothesis put forward above.

The manner by which the cushions are formed and the significance of the elastic tissue reduplication is also of great interest. A close relationship between muscle and elastic tissue changes in arterial walls was observed by Jores (1903, quoted by McMeans, 1915). Jores believed that splitting of elastic tissue is brought about by a mechanical forcing apart of the fibres due to an overgrowth of smooth muscle cells and that a muscle cell hyperplasia always precedes the splitting of elastic fibres. That muscle cells can migrate from the media into the intima through gaps in the internal elastica was shown by Gross, Epstein, and Kugel (1934) in their study of adult coronary arteries. More recently Altschul (1950) also described smooth muscle migration from the media into the intima in experimental and human arteriosclerosis and observed that these migrating cells were radially orientated.

Some support is given to these observations by the present study. The primary role of the media in the formation of intimal cushions has been stressed and the radial rotation of medial muscle fibres both in the formation of longitudinal muscle bands and of fibres which are apparently traversing fenestrae in the internal elastica has been 0 described. Even in the earliest stages of splitting of this membrane evidence of muscles migrating can be found (Fig. 6), suggesting a causal relationship between the two events. On this basis, too, an interpretation can be made of the sequence of events in the formation of a cushion. The primary factor is the pulsatile stress which stimulates a local migration and longitudinal reorientation of the circular muscle of the media with the formation of a simple longitudinal band. If the stress is marked, then there must also be a thickening or hypertrophy of the wall at this point. This is accomplished by a migration of muscle through the internal elastic lamina which is accompanied by a splitting of this membrane and, if sufficiently marked, results in a gross musculo-elastic thickening of the intima (Figs. 3 and 4). The character of the splitting of the internal elastica, whether coarse or fibrillary, depends perhaps on the rate of the process, mild stresses with gradual hypertrophy resulting in an indolent and coarse splitting of the lamina, and severe ones with a rapid intimal thickening and a fine fibrillary mesh.

When the development of these cushions is viewed in this light it is clear that the elastic tissue changes associated with them are not degenerative in nature but are part of a remodelling process. It is difficult to be certain of the significance of the fibrous tissue which is prominent in some cushions. In this connexion the work of Burton et al. (1955) is of interest. These workers have suggested that elastin is a derivative of collagen and showed that, by suitable treatment, collagen can be converted in vitro into a material which is virtually identical with elastin. If such a close relationship does exist, then it would hardly be surprising that, especially in those cushions with a fine elastic mesh and a relatively quick forma- 
tion of elastic fibrils, there should sometimes be an appreciable amount of connective tissue.

The significance of the occasional deposits of lipoid is also uncertain. Its presence was not related to the presence or absence of fibrous tissue. This feature was also noted by Moon. It seems probable that it is but a manifestation of local stress and is akin to the fatty flecking which is seen so commonly around the branches of the aorta and in the muscular arteries of children.

I wish to thank Professor J. H. Biggart for his help and encouragement during the course of this work. Mr. D. Mehaffey was responsible for the photographs.

\section{REFERENCES}

Altschul, R. (1950). Selected Studies on Arteriosclerosis, p. 50 . Thomas, Springfield, Illinois.

Anderson, W. A. D. (1953). Pathology, 2nd ed., p. 477. Mosby. St. Louis.

Burton, D., Hall, D. A., Keech, M. K., Reed, R., Saxl, H., Tunbridge, R. E., and Wood, M. J. (1955). Nature, Lond., 176, 966.

Dock, W. (1946). J. Amer. med. Ass., 131, 875.

Duguid, J. B. (1926). J. Path. Bact., 29, 371.

Fangman, R. J., and Hellwig, C. A. (1947). Amer. J. Path., 23, 901.

Gross, L., Epstein, E. Z., and Kugel, M. A. (1934). Ibid., 10, 253

Gross, L., Epstein, E. Z., and Kugel, M. A. (1934) Ibid., 10, 253.

Levene, C. I. (1956). J. Path. Bact., 72, 79.

McMeans, J. W. (1915). J. med. Res., 32, 377

Minkowski, W. L. (1947). Amer. J. med. Sci., 214, 623.

Moon, H. D. (1957). Circulation, 16, 263.

Moschcowitz E. (1942). Vascular Sclerosis, p. 129. Oxford University Press, New York.

Sodeman, W. A. (1956). Pathologic Physiology, 2nd ed., p. 291. Saunders, Philadelphia and London. 